\title{
Optimal distributed generation allocation in radial distribution systems considering customer-wise dedicated feeders and load patterns
}

\author{
Neeraj KANWAR ${ }^{1}$, Nikhil GUPTA ${ }^{1}$, K. R. NIAZI ${ }^{1}$, Anil SWARNKAR ${ }^{1}$
}

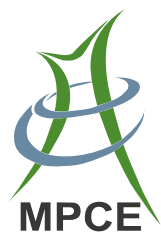

\begin{abstract}
Distribution system planners usually provide dedicated feeders to its different class of customers, each of whom has its own characteristic load pattern which varies hourly and seasonally. A more realistic modeling should be devised by considering the daily and seasonal variations in the aggregate load patterns of different class of customers. This paper addresses a new methodology to provide integrated solution for the optimal allocation of distributed generations and network reconfiguration considering load patterns of customers. The objectives considered are to maximize annual energy loss reduction and to maintain a better node voltage profile. Bat algorithm (BA) is a new bio-inspired search algorithm which has shown an advance capability to reach into the promising region, but its exploration is inadequate. The problem is solved by proposing the improved BA (IBA). The proposed method is investigated on the benchmark IEEE 33-bus test distribution system and the results are very promising.
\end{abstract}

Crosscheck date: 19 October 2015

Received: 8 July 2015 / Accepted: 19 October 2015 / Published online: 16 November 2015

(C) The Author(s) 2015. This article is published with open access at Springerlink.com

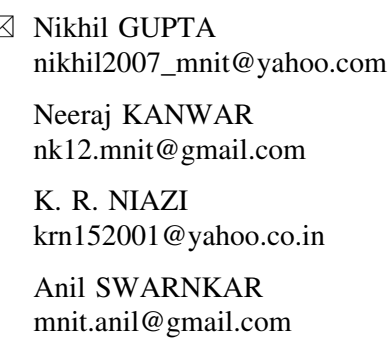

1 Department of Electrical Engineering, Malaviya National Institute of Technology, Jaipur, India
Keywords Load pattern, Distributed generations, Distribution network reconfiguration, Improved bat algorithm(IBA), Smart grid

\section{Introduction}

The electric power industries have witnessed many reforms in recent years. The rise of smart grid is a boon not only to society as a whole but to all who are involved in the electric power industry, its customers, and its stakeholders [1]. The existing distribution systems are moving towards smart distribution systems to achieve larger socio-economic and other non-tangible benefits. Distributed generations (DGs) are now becoming essential components for realizing the concept of smart distribution systems on account of economic viability and strict environmental laws. Optimal DG placement can improve network performance in terms of better node voltage profiles, reduce flows and system losses and improve power quality and reliability of supply, but inappropriate DG placement may increase system losses and network capital and operating costs [2]. Due to unbundling rules, distribution network operators (DNOs) cannot invest in generation facilities and are meant to provide DG owners with cost-effective connection means, irrespective of the technology or geographical location [3]. Therefore, future distribution networks with the high DG penetration to be planned and operated in order to improve their efficiency [4]. The optimal allocation of DG units requires the determination of their optimal sizing and sites to achieve desired objectives while satisfying several constraints. Thus, DG optimal allocation is a nonlinear complex combinatorial constraint optimization problem and is one of the most important and challenging task towards realizing smart grid objectives. 
Recently, some researchers have shown fruitful impact of optimal DG allocation on the performance of distribution systems using meta-heuristic techniques by considering a variety of technical or techno-economic objectives. Ref. [5] employed harmony search algorithm with differential operator to install multiple DG units with an objective of minimizing active power loss and improving voltage profile. In Ref. [6], the problem is solved for congestion management considering economic and financial issues. Two different methods, based upon locational marginal price and congestion rent, are proposed in order to mitigate transmission congestion and to increase security in restructured power systems. Ref. [7] presented a quasioppositional teaching learning based optimization methodology to simultaneously optimize power loss, voltage stability index and voltage deviation of radial distribution network. Ref. [8] proposed a combined imperialist competitive algorithm (ICA)-genetic algorithm (GA) method in which first, ICA is used to find the allocations of DGs and then GA operators are employed to refine these solutions. Ref. [9] proposed a multi-objective index-based approach for optimally determining the size and location of multi-DG units in distribution systems using particle swarm optimization (PSO) with different load models. A novel approach based on cuckoo search is applied for optimal DG allocation to improve voltage profile and reduce power loss of the distribution network in [10]. In most of the reported articles DGs have been treated as essentially the source of active power generations. However, from a purely operational perspective, DG plants could also be encouraged to provide reactive power support if that is of concern for the DNO or the regional transmission network [3]. Ref. [11] employed artificial bee colony algorithm to solve optimal DG allocation problem where DGs are capable to generate both active and reactive power. They have shown a substantial improvement in voltage profile and power loss reduction. Very recently, heuristic curve fitting technique [12], PSO [13] and analytical approach $[14,15]$ are also reported to investigate this problem with different types of DG units. It has been concluded in [14] that among different DG types, the DG capable of delivering both real and reactive power reduces losses more than that of DG capable of delivering real power only.

Distribution network reconfiguration (DNR) is one of the effective operational strategies to achieve high performance of distribution systems. Distribution networks are reconfigured frequently with changing operating conditions. Smart grid requires integrated solutions that reflect coexistence of strategies to achieve higher energy efficiency and good quality power supply. Some researchers [16-20] have employed DNR in conjunction with the optimal allocation of DGs and acknowledged that this strategy is more useful. But this approach is not realistic since the solution obtained can demand an alteration in both network topology and sites of DGs. In practice, the network topology can be altered, but not the locations of DGs with variations in load demand. Ref. [21] employed different combinatorial strategies of DGs allocation and DNR. But, according to their strategy, DNR should be carried out before DG addition. In fact, the problem of planning horizon should be dealt before optimizing any operational strategies, otherwise it may leads to erroneous results. Moreover, they have not taken into consideration the node voltage deviations and thermal limits of distribution feeders in the problem formulation, although both DNR and DGs can play crucial role to decide the voltage profiles and line flows of distribution systems.

The load demand of the system is a major source of uncertainty in power system planning [22], and is originated owing to the diversity of load demand among different class of customers. In practical situations, loads are mixtures of different load types, depending on the nature of the area being supplied, therefore, a load class mix of residential, industrial, and commercial loads is to be investigated too, in which every bus of the system has a different type of load connected to it [9]. Usually, distribution system planners provide dedicated feeders to its different class of customers, each of whom has its own characteristic load pattern which varies hourly and seasonally. A more realistic modeling should be devised by considering the daily and seasonal variations in the aggregate load patterns of different class of customers. Therefore, the modeling of annual load profile should be made more realistic by considering customer-wise dedicated feeders and their characteristic load patterns while dealing with resource allocation problems of distribution systems.

Bat Algorithm (BA) is a new bio-inspired optimization technique proposed in [23]. The algorithm is inspired by the social behavior of bats and the phenomenon of echolocation to sense distance. BA mimics the variations of pulse emission rates and loudness of bats when searching for prey and has shown a typical quick start. It is simple, robust, easy to implement and also significantly faster than other optimization techniques. It has been successfully applied to solve diverse engineering optimization problems such as power system stabilizer [24], uninhabited combat air vehicle (UCAV) path planning [25], economic dispatch [26], load frequency control [27], planning the sports training [28], fuel arrangement optimization of reactor core [29], feature selection [30], etc. The algorithm obtained good results when dealing with lower-dimensional optimization problems, but may become problematic for higher-dimensional problems because it tends to converge very fast initially [31]. The standard BA is usually quick at 
the exploitation of the solution though its exploration ability is relatively poor [25].

In the light of the above discussion, an improved BA (IBA) algorithm is proposed for optimal allocation of DGs in distribution systems to maximize annual energy loss reduction and to improve node voltage profiles. A realistic methodology has been adopted to formulate the problem considering dedicated feeders to various classes of customers and also considering the daily and seasonal variations in their characteristic load patterns. The distribution network is reconfigured for each operating state after placing optimal DGs which are capable to inject both active and reactive power. Some modifications are suggested in IBA to enhance the convergence, accuracy and efficiency of the standard BA. The proposed method is investigated on the benchmark IEEE 33-bus test distribution system and the application results obtained are very promising.

\section{Load patterns of customers}

Several earlier works reported have employed threestepped load profile of distribution system for its piecewise linearization. However, power demand of a distribution network is stochastic in nature and is primarily depends upon the category of customers. Each class of customers has its characteristic load pattern which varies hourly and seasonally. Distribution system planners usually provide dedicated feeders for each class of customers. In this paper, distribution feeders are assumed to be divided into residential, industrial and commercial categories. Therefore, the diversity available among different customers is taken into account while determining aggregate annual load profile of the distribution system.

For each category, the aggregate daily load pattern of the customer is usually approximated by piecewise linearization into three or four levels. However, they may have different load durations. Therefore, the system load profile for a given season may have several load levels as shown in Fig. 1. The figure shows that there are total nine load levels for an individual season called spring-fall season. Similarly, the load pattern for other seasons, e.g. summer and winter seasons can be determined by considering seasonal variations in the demand of each category of customers. The aggregate annual load profile of the system can be obtained by the summation of load patterns being generated for all seasons of the year. For the illustration, the annual load profile will be composed of 27 load levels while considering three seasons.

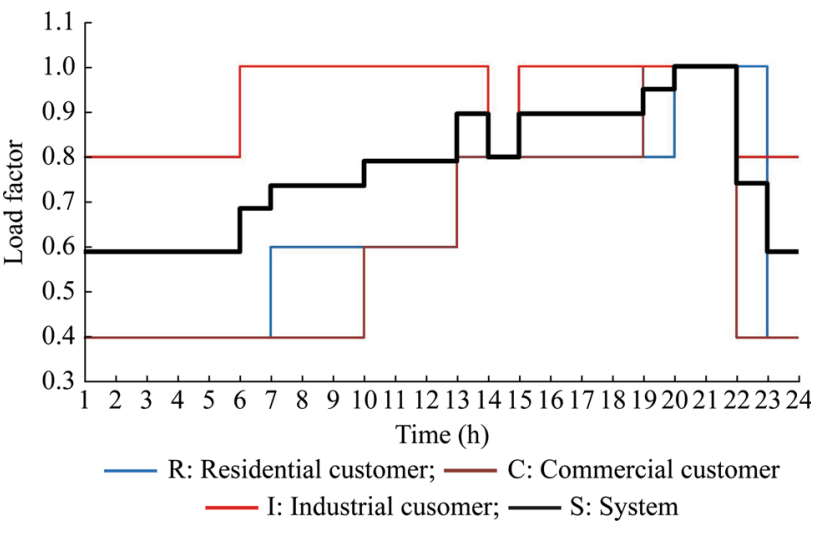

Fig. 1 Aggregate daily load patterns of customers and the system

\section{Problem formulation}

The problem is formulated by considering dedicated feeders being assigned to various customers and by considering hourly and seasonal variations in the load patterns of each class of customers to determine the annual load profile of the system. However, whatever the particular driver is for a DNO, e.g., to allow the connection of more DG capacity, to reduce energy losses, or to increase network reliability, these DG planning tools must take into account essential network constraints such as voltage and thermal limits [3]. The objective function $O$ is therefore designed to minimize annual energy loss reduction while enhancing node voltage and feeder current profiles using penalty function approach as below.

$O=B\left(E_{\text {loss }}^{\text {base }}-E_{\text {loss }}^{\mathrm{DG}}\right)$

where $E_{\text {loss }}^{\text {base }}$ and $E_{\text {loss }}^{\mathrm{DG}}$ are the annual energy losses without and with DG placement and can be determined using following equation assuming piecewise linearized load profile of the system.

$E_{\text {loss }}=\sum_{s=1}^{N_{s}} D_{s} \sum_{j=1}^{L} H_{j, s}\left(\sum_{r=1}^{N_{r}} P_{\text {loss }}^{r}+\sum_{i=1}^{N_{i}} P_{\text {loss }}^{i}+\sum_{c=1}^{N_{c}} P_{\text {loss }}^{c}\right)$

where $P_{\text {loss }}^{r}, P_{\text {loss }}^{i}, P_{\text {loss }}^{c}$ are the power losses in $r^{\text {th }}$ residential, $i^{\text {th }}$ industrial and $c^{\text {th }}$ commercial feeder, respectively at $j^{\text {th }}$ load level of the $s^{\text {th }}$ season. $H_{j, s}$ and $D_{s}$ are the load duration for $j^{\text {th }}$ level of $s^{\text {th }}$ season and number of days in a season, respectively. $N_{s}$ denotes the number of seasons in a year and $N_{r}, N_{i}, N_{c}$ are the set of nodes lies along residential, industrial and commercial feeders, respectively. The penalty function $P F$ is defined as: 
$B=\sqrt{V_{p f} \times I_{p f}}$

where voltage and current penalty functions are given by

$V_{p f}=\frac{1}{1+\left(\operatorname{Max}\left(\Delta V_{r, j, s}, \Delta V_{i, j, s}, \Delta V_{c, j, s}\right)\right)} \quad \forall i \in N, \forall j \in L$

$I_{p f}=\frac{1}{1+\left(\operatorname{Max}\left(\Delta I_{r, j, s}, \Delta I_{i, j, s}, \Delta I_{c, j, s}\right)\right)} \quad \forall i \in N, \forall j \in L$

where $\Delta V_{r, j, s}, \Delta V_{i, j, s}, \Delta V_{c, j, s}$ are the maximum node voltage deviations of $r^{\text {th }}$ residential node, $i^{\text {th }}$ industrial node and $c^{\text {th }}$ commercial node at $j^{\text {th }}$ load level of the $s^{\text {th }}$ season. Similarly, $\Delta I_{i, j, s}, \Delta I_{i, j, s}, \Delta I_{c, j, s}$ are the maximum branch current deviations of $r^{\text {th }}$ residential node, $i^{\text {th }}$ industrial node and $c^{\text {th }}$ commercial node at $j^{\text {th }}$ load level of the $s^{\text {th }}$ season. $N$ and $L$ denote the set of system nodes and load levels. Node voltage deviation and feeder current deviations at $r^{\text {th }}$ residential feeder for $j^{\text {th }}$ load level of the $s^{\text {th }}$ season are defined as:

$\Delta V_{r, j, s}=\left\{\begin{array}{cc}1-\left|V_{r, j, s}\right| & V_{\operatorname{minS}}<V_{r, j, s}<V_{\min } \\ 0 & V_{\min } \leq V_{r, j, s} \leq V_{\max } \\ 10^{10} & \text { else }\end{array}\right.$

$\Delta I_{r, j, s}=\left\{\begin{array}{cc}I_{\operatorname{max~S}}^{r}-I_{r, j, s} & I_{\max }^{r}<I_{r, j, s} \leq I_{\operatorname{max~S}}^{r} \\ 0 & I_{r, j, s} \leq I_{\max }^{r} \\ 10^{10} & \text { else }\end{array}\right.$

where minimum and maximum bounds for node voltages and feeder currents are denoted by the subscript min and max, respectively. $V_{\text {mins }}$ denotes specified node voltage suggested for better capacity allocation with the due consideration for network reconfiguration and $I_{\max }$ is thermal limit of feeders after considering maximum tolerance, usually taken as $10 \%$ above the rated value. Similarly, $\Delta V_{i, j, s}, \Delta V_{c, j, s}, \Delta I_{i, j, s}$ and $\Delta I_{c, j, s}$ can also be defined. The different constraints used to solve $O$ are defined as below.

\subsection{Power flow equations}

The sum of the power purchased from utility grid and the total power generated by the different sources in the distribution system must be balanced by the local load demand and the power loss in the lines. For a radial network, a set of recursive equations are used to model the power flow in the network as shown by (8)-(12).

$$
\begin{array}{ll}
P_{n+1}=P_{n}-R_{n} \frac{P_{n}^{2}+Q_{n}^{2}}{V_{n}^{2}}-p_{n+1} \quad \forall n \in N \\
Q_{n+1}=Q_{n}-X_{n} \frac{P_{n}^{2}+Q_{n}^{2}}{V_{n}^{2}}-q_{n+1} \quad \forall n \in N
\end{array}
$$

$$
V_{n+1}^{2}=V_{n}^{2}-2\left(R_{n} P_{n}+X_{n} Q_{n}\right)+\left(R_{n}^{2}+X_{n}^{2}\right) \frac{P_{n}^{2}+Q_{n}^{2}}{V_{n}^{2}}
$$

$\forall n \in N$

$$
\begin{array}{ll}
p_{n+1}=p_{n+1}^{L}-p_{n+1}^{\mathrm{DG}} & \forall n \in N \\
q_{n+1}=q_{n+1}^{L}-q_{n+1}^{\mathrm{DG}} & \forall n \in N
\end{array}
$$

The active and reactive nodal power flows are expressed by the upper case notations $P$ and $Q, R$ and $X$ denotes resistance and reactance of the feeder whereas the active and reactive power injections are denoted by the lower case notations $\mathrm{p}$ and $\mathrm{q}$, respectively. $V$ denotes the voltage of the bus of the distribution system.

\subsection{Node compensation limit}

The total complex power injected by DG at each node must be within their permissible range as defined by (13).

$S_{\mathrm{DG}, \min } \leq S_{\mathrm{DG}, n} \leq S_{\mathrm{DG}, \max } \quad \forall n \in N$

\subsection{System compensation limit}

The sum of total complex power injected by DGs at all candidate nodes should be less than nominal complex power demand $S_{\mathrm{D}}$ of the distribution system. For Loc number of candidate locations of DGs the system compensation limit is defined as

$\sum_{n=1}^{\text {Loc }} S_{\mathrm{DG}, n} \leq S_{\mathrm{D}} \quad \forall n \in N$

Equation (15) prohibits repetition of candidate sites for DGs. Discrete dispatches of DGs are modeled as (16) for $\Delta S$ unit size of DG.

$N_{\mathrm{DG}, a} \neq N_{\mathrm{DG}, b} \quad a, b \in N$
$S_{\mathrm{DG}} \leq k_{d} \Delta S \quad k_{d}=0,1,2, \ldots, n d g$

where $n d g$ denotes DG penetration limit for each system node. First optimizing (1), the solution obtained provides the optimal sizes and sites of DGs, while considering the annual load profile. Next, (1) is optimized again, but for each load level separately, to determine the optimal power dispatches of these installed DGs. However, the sites for DGs are kept freeze and their sizing is restricted to that provided by the obtained solution. The additional constraint defined for optimal dispatches of DGs is given below:

$S_{\mathrm{DG}}=k_{\mathrm{md}} \Delta S \quad k_{\mathrm{md}}=0,1,2, \ldots, S_{\mathrm{DG}} / \Delta S$

The distribution networks are reconfigured frequently to optimize energy efficiency and to maintain power quality. Therefore, the distribution network is reconfigured at each load level after placing respective dispatches of DGs. The 
reconfiguration problem is formulated to minimize feeder power losses while satisfying various network operational constraints. The mathematical formulation for the network reconfiguration problem to minimize power losses is considered as:

Min $P_{\text {loss } . j}=\sum_{n=1}^{E} R_{n} \frac{P_{n, j}^{2}+Q_{n, j}^{2}}{\left|V_{n, j}\right|^{2}} \quad \forall n \in N, \forall j \in L$

subject to the following constraints.

\subsection{Radial topology constraint}

The reconfigured network topology must be radial, i.e. with no closed path. Therefore, for the $r^{\text {th }}$ radial topology,

$\Phi_{j}(r)=0 \quad \forall j \in L$

\subsection{Node voltage constraint}

A hard voltage constraint is employed during the network reconfiguration as it is one of the important network operation strategies. All node voltages of the system must be maintained within the following limits during the optimization process.

$V_{\min } \leq V_{n, j} \leq V_{\max } \quad \forall n \in N, \forall j \in L$

The radial constraint imposes the biggest hurdle while solving the problem of network reconfiguration. In the present work, the codification proposed in [32] is used to solve the problem. This is a rule-based codification to check and correct infeasible radial topologies. According to this codification, three following rules are formed which are based on graph theory to identify and correct infeasible individuals whenever appeared in the computational process.

1) Each candidate switch must belong to its corresponding loop vector.

2) Only one candidate switch can be selected from one common branch vector.

3) All the common branch vectors of a prohibited group vector cannot participate simultaneously to form an individual. The definitions of loop vector, common branch vector and prohibited group vector may be referred from [32].

\section{Bat algorithm}

Bats are fascinating animals. They have advanced capability of echolocation [23]. In BA, the velocity and position of each bat are updated with iterations as in PSO. However, an intensive local search is also combined which is very similar to simulated annealing. It is governed by the loudness and pulse emission rate assigned to each bat and are allowed to vary with iterations. The different phases used in standard BA can be summarized as below.

\subsection{Random fly}

Each bat is defined by its position $x_{i}(t)$, velocity $v_{i}(t)$, frequency $f_{i}$, loudness $A_{i}(t)$ and the pulse emission rate $r_{i}(t)$ at the time step $t$ in a $d$-dimensional problem search space

$f_{i}=f_{\min }+\left(f_{\max }-f_{\min }\right) \times \beta$

$v_{i}(t)=v_{i}(t-1)+\left(x_{i}(t)-x^{*}\right) \times f_{i}$

$x_{i}(t)=x_{i}(t-1)+v_{i}(t)$

where $\beta$ is a random number in the range $[0,1]$.

\subsection{Local random walk}

For the local search part, once a solution is selected among the current best solutions $x^{*}$, a new solution for each bat is generated locally using random walk as defined below:

$x_{\text {new }, i}=x_{\text {old }, i}+\varepsilon<A(t)>$ rand $>r_{i}(t)$

where $\varepsilon$ is a scaling factor in the range $[-1,1]$ and rand is a random number in the range $[0,1] .<A(t)>$ is the average loudness of all bats. The loudness and the pulse emission rate of each bat are updated with iteration using following relations.

$A_{i}(t+1)=\alpha A_{i}(t)$

$r_{i}(t+1)=r_{i}(0)\left[1-\mathrm{e}^{-\gamma t}\right]$

where $\alpha$ and $\gamma$ are constant, each of them is usually taken as 0.9 [23].

\section{Proposed IBA}

BA has very good exploitation potential, but the exploration potential is inadequate. Therefore, it has legacy of lack in diversity which eventually leads to local trappings and thus the convergence degrades. Therefore IBA is proposed as an optimizing tool after suggesting several modifications in the standard BA algorithm, viz. revised loudness and pulse emission rate, improved local random walk and diversity to improve performance of the standard BA.

\subsection{Revised loudness and pulse emission rates}

The loudness and pulse emission rates essentially provide a mechanism for automatic control and auto zooming 
into the region with promising solutions [33]. The gradual switching of exploration to exploitation is governed by the relative values assigned to loudness and the pulse emission rate. In BA, both are allowed to vary too quickly, which may lead to stagnation after some initial stage [33]. Therefore, the loudness and pulse emission rates are revised as below.

$A_{i}(t)=\alpha^{t} A_{i}$

$r_{i}(t)=1-A_{i}(t)$

where $\alpha=\left(1 / 2 A_{i}\right)^{1 /\left(k * i r_{\max }\right)}$, and $k$ is the desired fraction of maximum iteration count $i t r_{\max }$ at which loudness and pulse emission rate equalizes. In this way, these two parameters become self-adjustable.

\subsection{Improved local random walk}

In standard $\mathrm{BA}$, the term $\varepsilon<A(t)>$ remains ineffective for most of the bats, especially during later portion of the computational process while considering $\varepsilon \in[-1,1]$ and the initial loudness $A \in[0,1]$. As a consequence, the local random walk also becomes ineffective while solving an optimization problem with discrete decision variables. This adversely affects the exploitation potential of the algorithm. Therefore, an improved local random walk of the best bat is proposed as described below.

First of all, $M_{c}$, number copies of the best bat are generated and each of them is mutated over the randomly selected $M_{n}$ dimensions in the range $[-M, M]$. The choice of $M_{c}$, is decided by the usual tradeoff between accuracy and CPU time of the algorithm. The higher the value of $M_{c}$, the better will be the exploration, but at the cost of more CPU time. Usually, $M_{c}(>4)$ provides satisfactory results. Higher value of $M_{n}(>0.6)$ causes much higher diversity and may diverge solutions. With $M>2$, it will cause unnecessarily larger search space for the local random walk. The best bat is replaced if a better bat is available after this improved local random walk.

\subsection{Diversity}

BA has inheritance in lack of diversity. This may causes poor exploration as the bats are exploring extensively the region in the near vicinity of the current best bat. The mutation operator is capable to identify unexplored areas of the search space. Since BA has very strong exploitation potential, a very high mutation rate is desired to makes it effective. Therefore, the algorithm is reinitialized using single-point random mutation. The Fitness of all mutated bats is evaluated and the current best bat is updated if the better one is found.

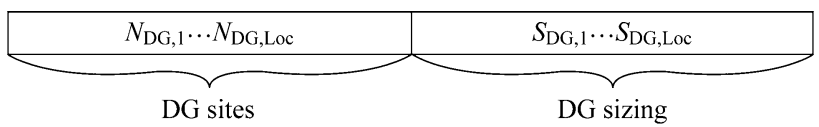

Fig. 2 Structure of Individuals

\subsection{Structure of individuals}

The for the proposed method is shown in Fig. 2 which is composed of candidate nodes $N_{\mathrm{DG}}$ and sizing $S_{\mathrm{DG}}$ for the candidate DGs. The candidate nodes and DG sizing are selected randomly within their respective predefined bounds as described by (13)-(15). When determining optimal power dispatches of the installed DGs, for each load level, the algorithm again runs with the same structure of individuals. However, the DGs locations are now freeze to those values that obtained by the optimal solution. Also the limit of sizing is restricted to the installed capacities that provided by the optimal solution.

\subsection{Elitism and termination criterion}

Elitism is used to preserve the best bat during the evolutionary process. The termination criterion may be selected by defining maximum iteration count or accuracy of solutions, etc. However, in the proposed method, when either the maximum iteration count is exhausted or all candidate solutions acquire the same fitness, the iterative computation stops.

The Pseudo code of the proposed IBA is presented as below:

Objective function $O$

Initialize the bat population $x_{i}, v_{i}$ and $f_{i, d} i=1,2, \ldots$,

$N_{b}, d=1,2, \ldots, D$

Initialize pulse rate $r_{i}$ and loudness $A_{i}$

While $\left(t<i t r_{\max }\right)$

Generate new solutions by adjusting frequency, and updating velocities and locations/solutions

if $\left(\right.$ rand $>r_{i}$ )

Select the current best solution

Generate a local solution $\left(F_{1}\right)$ around the current best solution

else if

Make $M_{c}$ copies of the current best solution

Apply improved local random walk

Select best solution $\left(F_{2}\right)$ among $M_{c}$ solutions after single-point random mutation

Select best solution $F$ out of $F_{1}$ and $F_{2}$

end if

Generate a new solution by flying randomly

if (rand $<A_{i}$ and $\left.O<F\right)$ ) then

Accept the new solutions 
Increase $r_{i}$ and reduce $A_{i}$

end if

Rank the bats and find the current best $x^{*}$

$t=t+1$;

Apply diversity and preserve elite solution

end while

Post-processing the results and visualization.

\section{Simulation results and discussion}

The proposed method is applied to IEEE 33-bus test distribution system [34]. The initial data of this test system can be referred from Table 1 . The hourly and seasonal variations in the load factors considered for different varieties of customers are presented in Table 2. In the table, $\mathrm{L}, H_{j}$, R, I, and C denote load level, load duration in hours, load factor for residential, industrial and commercial customer in p.u., respectively. The table shows load factors and corresponding load durations for the aggregate daily load patterns of the given season-wise customers. The load levels 1-9, 10-18 and 19-27 are used for spring/fall, winter and summer seasons, respectively. Table 3 shows design parameters selected for this problem.

The best solution is obtained after 100 trial runs of proposed IBA method. The optimal sites of DGs to be installed in the distribution network are 13, 24 and 30, whose corresponding optimal sizes are $565 \mathrm{kVA}, 1049 \mathrm{kVA}$ and $958 \mathrm{kVA}$, respectively. After placing optimal DGs, their optimal active and reactive power dispatches are determined at each load level separately as shown in Table 4. The distribution network is reconfigured at each load level after optimally placing DGs. The optimal network configurations are also shown in the table. After providing this integrated solution, feeder power losses and minimum node voltage are determined for each load level as shown in Figs. 3 and 4, respectively.

It can be observed from these figures that the power losses are substantially reduced and the minimum node voltage is significantly enhanced using optimal DG

Table 1 Initial data of IEEE 33-bus system

\begin{tabular}{ll}
\hline Particular & Value \\
\hline Line Voltage $(\mathrm{kV})$ & 12.66 \\
Nominal Active Demand (kW) & 3715 \\
Nominal Reactive Demand (kVA) & 2300 \\
Base Configuration & 33 to 37 \\
Residential Feeders & $1-15$ \\
Industrial Feeders & $22-29$ \\
Commercial Feeders & $16-21,30-33$ \\
\hline
\end{tabular}

Table 2 Load factors and load duration for different seasons

\begin{tabular}{llllllllll}
\hline $\mathrm{L}$ & $\mathrm{R}$ & $\mathrm{I}$ & $\mathrm{C}$ & $H_{j}$ & $\mathrm{~L}$ & $\mathrm{R}$ & $\mathrm{I}$ & $\mathrm{C}$ & $H_{j}$ \\
\hline 1 & 0.40 & 0.80 & 0.40 & 7 & 15 & 0.48 & 0.48 & 0.48 & 1 \\
2 & 0.40 & 1.00 & 0.40 & 1 & 16 & 0.48 & 0.60 & 0.60 & 1 \\
3 & 0.60 & 1.00 & 0.40 & 3 & 17 & 0.60 & 0.60 & 0.60 & 2 \\
4 & 0.60 & 1.00 & 0.60 & 3 & 18 & 0.60 & 0.48 & 0.24 & 1 \\
5 & 0.80 & 1.00 & 0.80 & 5 & 19 & 0.56 & 1.12 & 0.56 & 7 \\
6 & 0.80 & 0.80 & 0.80 & 1 & 20 & 0.56 & 1.40 & 0.56 & 1 \\
7 & 0.80 & 1.00 & 1.00 & 1 & 21 & 0.84 & 1.40 & 0.56 & 3 \\
8 & 1.00 & 1.00 & 1.00 & 2 & 22 & 0.84 & 1.40 & 0.84 & 3 \\
9 & 1.00 & 0.80 & 0.40 & 1 & 23 & 1.12 & 1.40 & 1.12 & 5 \\
10 & 0.24 & 0.48 & 0.24 & 7 & 24 & 1.12 & 1.12 & 1.12 & 1 \\
11 & 0.24 & 0.60 & 0.24 & 1 & 25 & 1.12 & 1.40 & 1.40 & 1 \\
12 & 0.36 & 0.60 & 0.24 & 3 & 26 & 1.40 & 1.40 & 1.40 & 2 \\
13 & 0.36 & 0.60 & 0.36 & 3 & 27 & 1.40 & 1.12 & 0.56 & 1 \\
14 & 0.48 & 0.60 & 0.48 & 5 & - & - & - & - & - \\
\hline
\end{tabular}

Table 3 Design parameters for optimally placing DGs

\begin{tabular}{ll}
\hline Particular & Value \\
\hline$V_{\text {mins }} / V_{\min } / V_{\max }$ (p.u.) & $0.9 / 0.95 / 1.05$ \\
$S_{\mathrm{DG}}(\mathrm{MW})$ & 2 \\
$P F_{\mathrm{DG}}$ & 0.85 \\
$S_{\mathrm{D}}(\mathrm{kVA})$ & 7000 \\
$N_{\mathrm{DG}}$ & $1-33$ \\
$\Delta S(\mathrm{kVA})$ & 1 \\
Loc & 3 \\
$I_{\max }^{n}(n)$ & $400(1,2), 250(3-5,18-20,22-29)$, \\
$f_{\min } / f_{\max }$ & $150(6-17,21,30-37)$ \\
$\gamma$ & $0 / 2$ \\
$M_{c}$ & 0.9 \\
$M_{n}$ & 5 \\
$M$ & 0.6 \\
\hline
\end{tabular}

allocation. The performance of the distribution system is further enhanced by network reconfiguration. It happens at all load levels.

The annual energy loss, percentage annual energy loss reduction and the minimum node voltage among all the 27 load levels considered is presented in Table 5. It can be observed from the table that annual energy loss reduction is about $85 \%$ using DG allocation which is further enhanced to about $89 \%$ by network reconfiguration. Thus proposed integrated solution provides substantial reduction in annual energy losses occurred in distribution feeders. Also a better node voltage profile with a minimum node voltage of 0.9542 p.u. is obtained during the peak load hours. This shows that 
Table 4 Optimal dispatches of DGs and optimal configuration at different load levels (pf 0.85)

\begin{tabular}{lll}
\hline $\begin{array}{l}\text { Load } \\
\text { level }\end{array}$ & $\begin{array}{l}\text { Optimal dispatches } \\
\text { of DGs (kVA) }\end{array}$ & $\begin{array}{l}\text { Optimal } \\
\text { configuration }\end{array}$ \\
\hline 1 & $352,874,656$ & $7,8,9,21,25$ \\
2 & $359,1049,701$ & $7,8,9,21,26$ \\
3 & $480,1049,752$ & $7,9,21,25,36$ \\
4 & $528,1049,943$ & $7,9,21,22,25$ \\
5 & $565,1049,958$ & $7,8,23,34,35$ \\
6 & $565,1037,958$ & $7,8,25,34,35$ \\
7 & $565,1049,958$ & $7,8,28,34,35$ \\
8 & $565,1049,958$ & $7,9,28,34,35$ \\
9 & $565,1000,853$ & $7,8,9,21,37$ \\
10 & $215,525,390$ & $7,8,9,21,25$ \\
11 & $219,643,420$ & $7,9,21,26,36$ \\
12 & $293,659,439$ & $7,9,21,25,36$ \\
13 & $316,663,553$ & $7,8,9,21,25$ \\
14 & $413,686,705$ & $7,8,9,21,25$ \\
15 & $410,571,677$ & $7,8,9,21,25$ \\
16 & $438,692,821$ & $7,8,9,21,25$ \\
17 & $511,708,849$ & $7,8,9,21,25$ \\
18 & $428,591,469$ & $7,9,16,21,25$ \\
19 & $502,1049,938$ & $7,9,21,22,26$ \\
20 & $552,1049,958$ & $7,8,21,27,34$ \\
21 & $565,1049,958$ & $7,8,27,34,36$ \\
22 & $565,1049,958$ & $7,9,28,34,35$ \\
23 & $565,1049,958$ & $7,9,12,28,34$ \\
24 & $565,1049,958$ & $7,8,9,12,28$ \\
25 & $565,1049,958$ & $7,9,12,28,34$ \\
26 & $565,1049,958$ & $7,9,23,26$ \\
27 & $565,1049,958$ &
\end{tabular}

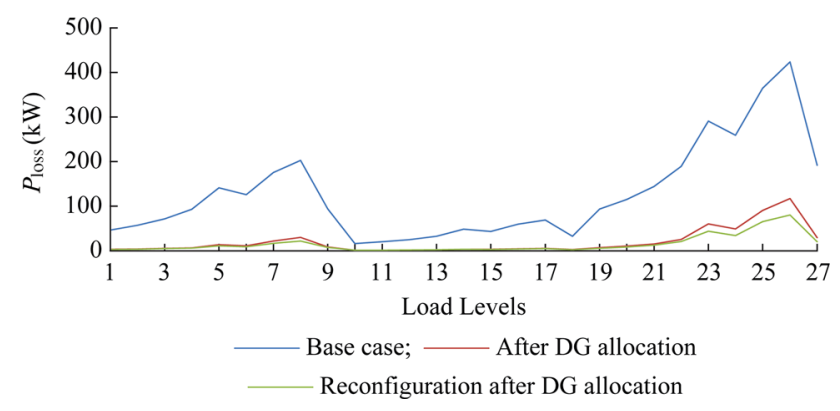

Fig. 3 Feeder power losses at different load levels

all node voltages maintained above the permissible limit during any load condition throughout the year.

A set of convergence characteristics for the best and mean fitness obtained after subsequently applying each modification in BA is shown in Fig. 5. In the figure, B1 represents the standard BA with revised loudness and pulse emission rates,

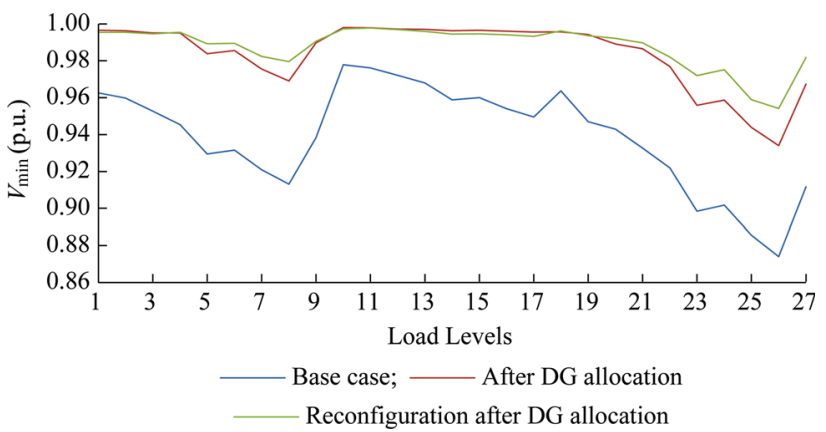

Fig. 4 Minimum node voltage at different load levels

Table 5 Comparison of network performance

\begin{tabular}{|c|c|c|c|}
\hline Particulars & Base case & $\begin{array}{l}\text { DG } \\
\text { allocation }\end{array}$ & $\begin{array}{l}\text { DG allocation } \\
\text { and network } \\
\text { reconfiguration }\end{array}$ \\
\hline Annual energy loss (kWh) & 984630.15 & 143,248 & 108,378 \\
\hline $\begin{array}{l}\text { Percentage annual energy } \\
\text { loss reduction }\end{array}$ & - & 85.45 & 88.99 \\
\hline $\begin{array}{l}\text { Minimum node voltage } \\
\text { (p.u.) }\end{array}$ & 0.8528 & 0.9340 & 0.9542 \\
\hline
\end{tabular}

B2 is B1 with improved local random walk and IBA, i.e. B2 with diversity. It can be observed from the figure that there is an improvement in the convergence of BA with each subsequent modification. As a result, there is a consistent improvement in the convergence of IBA. In IBA, the proposed loudness and pulse emission rates are made selfadaptive for all bats. This possibly enhances the exploration potential of BA to certain extent. As a result, IBA finds the promising region which keeps more patience than $\mathrm{BA}$. The application of improved local random walk is quite intense and it enables IBA to avoid many local trappings. The proposed diversity searches new solution points in the search space by re-initializing the algorithm. It plays a crucial role to cope against the extreme exploitation potential of BA. The diversity provides marked improvement in the convergence as can be seen from the figure where it shows a chain to avoid very fine local trappings.

Similar conclusions may be drawn from Fig. 6 which shows the comparison for mean convergence. It is noteworthy that the proposed diversity causes the mean fitness of the population to fluctuate and also tends to maintain it at a poor value during the evolutionary process though it enhances convergence for the best fitness. It happens as the population is re-initialized at the end of each iteration.

In order to further investigate the effectiveness of each modification in the proposed IBA, the statistical quality indices such as standard deviation (SD), coefficient of variation $(\mathrm{COV})$, error from the best $(\mathrm{EFB})$ and $\mathrm{CPU}$ time have been determined for the sampled solutions obtained 


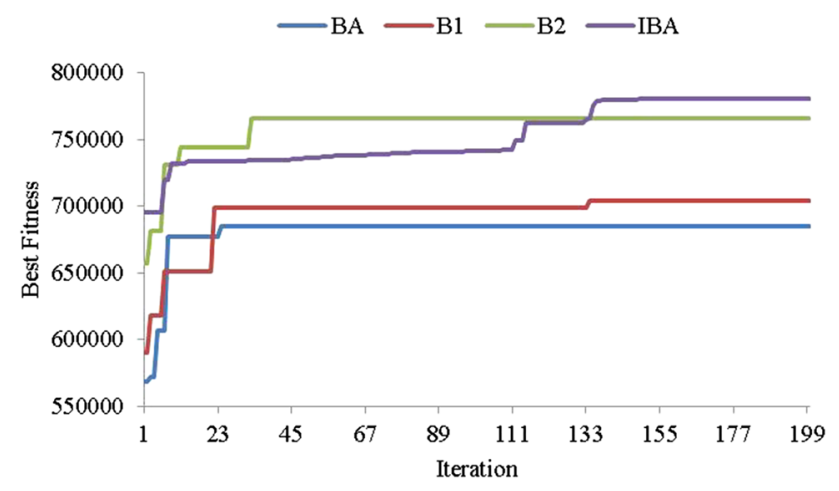

Fig. 5 Comparison of best fitness for BA and its proposed variants

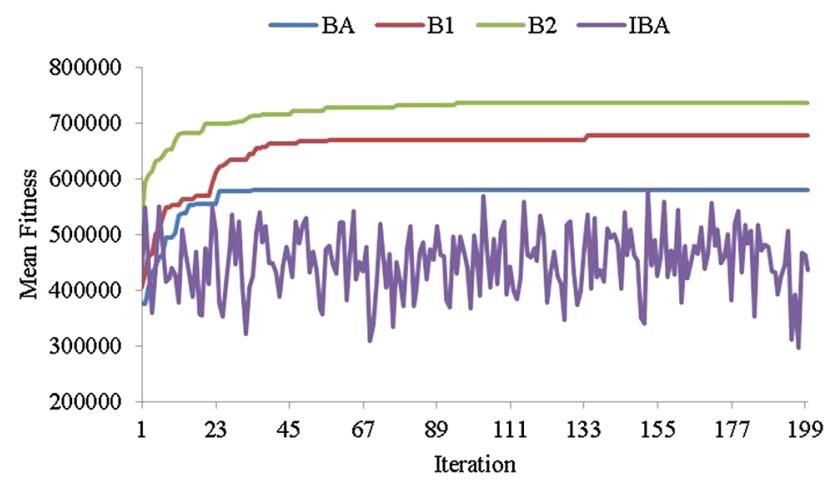

Fig. 6 Comparison of mean fitness for BA and its proposed variants

Table 6 Comparison of solution quality

\begin{tabular}{lcllc}
\hline Variants & SD & COV & EFB & CPU time (s) \\
\hline BA & 6447.69 & 0.82 & 1.48 & 58.16 \\
B1 & 4711.15 & 0.59 & 0.66 & 57.70 \\
B2 & 3995.24 & 0.51 & 0.65 & 57.74 \\
IBA & 2711.79 & 0.34 & 0.39 & 115.59 \\
GA & 7142.55 & 0.92 & 1.89 & 51.95 \\
PSO & 14522.02 & 1.90 & 3.11 & 57.66 \\
CSO & 9661.06 & 1.26 & 2.45 & 106.06 \\
\hline
\end{tabular}

by 100 independent trials of BA variants. The comparison results are presented in Table 6 . The smaller the value of these indices, the better the solution quality will be. The table shows a continuous improvement in the solution quality when all proposed modifications are sequentially applied in the standard BA. The table also presents the solution quality obtained while GA, PSO and cat swarm optimization (CSO) are also applied to this system with same population size and maximum iteration count as that of BA. It can be observed from the table that proposed IBA is more efficient and accurate than other related techniques. However, IBA is found to be more computationally demanding. It is obvious due to re-initialization of the population in order to maintain sufficient diversity which was essential to cope against the inherent tendency of BA to quickly approach in the promising region and then the algorithm trapped in local optima owing to extreme exploitation potential. Thus IBA generates better quality solutions, but at the cost of more CPU time.

\section{Conclusions}

This paper addresses a more realistic methodology for optimal allocation of DGs in radial distribution systems to maximize annual energy loss reduction while maintaining better node voltage profiles. A more realistic scenario of distribution network is considered by assuming dedicated feeders to its different class of customers and their specific load profiles that varies hourly and seasonally. The distribution network is reconfigured after placing optimal DGs. The application results in a standard test distribution system shows that a significant improvement in the desired objectives is achieved using proposed integrated approach for DG allocation and network reconfiguration. It is noteworthy that using the proposed approach, the peak load demand and its duration becomes more realistic on account of the diversity of load among various classes of customers. This reflects in the solution with positive sense. Nevertheless, more DG capacity installation or less improvement in node voltage profile, or both may be expected. The problem is solved by proposing an improved bat algorithm (IBA). The effectiveness of each suggested modifications in IBA has been thoroughly investigated. It has been observed that each modification is actively contributing towards improving the convergence, accuracy and efficiency of the algorithm. The proposed IBA can serve as a promising tool to solve hard combinatorial engineering optimization problems. The present approach can be extended for asset management in microgrids with DGs having intermittent power generations.

Open Access This article is distributed under the terms of the Creative Commons Attribution 4.0 International License (http:// creativecommons.org/licenses/by/4.0/), which permits unrestricted use, distribution, and reproduction in any medium, provided you give appropriate credit to the original author(s) and the source, provide a link to the Creative Commons license, and indicate if changes were made.

\section{References}

[1] El-Hawary ME (2014) The smart grid-state-of-the-art and future trends. Electr Power Compon Syst 42(3/4):239-250

[2] Georgilakis PS, Hatziargyriou ND (2013) Optimal distributed generation placement in power distribution networks: models, methods, and future research. IEEE Trans Power Syst 28(3):3420-3428

[3] Keane A, Ochoa LF, Borges CLT et al (2013) State-of-the-art techniques and challenges ahead for distributed generation 
planning and optimization. IEEE Trans Power Syst 28(2): 1493-1502

[4] Hemdan NGA, Deppe B, Pielke M et al (2014) Optimal reconfiguration of radial MV networks with load profiles in the presence of renewable energy based decentralized generation. Electr Power Syst Res 116:355-366

[5] Kollu R, Rayapudi SR, Sadhu VLN (2014) A novel method for optimal placement of distributed generation in distribution systems using HSDO. Int Trans Electr Energy Syst 24(4):547-561

[6] Afkousi-Paqaleh M, Fard AAT, Rashidinejad M (2010) Distributed generation placement for congestion management considering economic and financial issues. Electr Eng 92(6):193-201

[7] Sultana S, Roy PK (2014) Multi-objective quasi-oppositional teaching learning based optimization for optimal location of distributed generator in radial distribution systems. Int $\mathbf{J}$ Electr Power Energy Syst 63:534-545

[8] Moradi MH, Zeinalzadeh A, Mohammadi Y et al (2014) An efficient hybrid method for solving the optimal sitting and sizing problem of DG and shunt capacitor banks simultaneously based on imperialist competitive algorithm and genetic algorithm. Int $\mathrm{J}$ Electr Power Energy Syst 54:101-111

[9] El-Zonkoly AM (2011) Optimal placement of multi-distributed generation units including different load models using particle swarm optimization. IET Gener Transm Distrib 5(7):760-771

[10] Moravej Z, Akhlaghi A (2013) A novel approach based on cuckoo search for DG allocation in distribution network. Int $\mathrm{J}$ Electr Power Energy Syst 44:672-679

[11] Abu-Mouti FS, El-Hawary ME (2011) Optimal distributed generation allocation and sizing in distribution systems via artificial bee colony algorithm. IEEE Trans Power Deliv 26(4):2090-2101

[12] Naik SG, Khatod DK, Sharma MP (2013) Optimal allocation of combined DG and capacitor for real power loss minimization in distribution networks. Int J Electr Power Energy Syst 53:967-973

[13] Kansal S, Kumar V, Tyagi B (2013) Optimal placement of different type of DG sources in distribution networks. Int $\mathrm{J}$ Electr Power Energy Syst 53:752-760

[14] Hung DQ, Mithulananthan N (2013) Multiple distributed generator placement in primary distribution networks for loss reduction. IEEE Trans Ind Electron 60(4):1700-1708

[15] Elsaiah S, Benidris M, Mitra J (2014) Analytical approach for placement and sizing of distributed generation on distribution systems. IET Gener Transm Distrib 8(6):1039-1049

[16] Rao RS, Ravindra K, Satish K et al (2013) Power loss minimization in distribution system using network reconfiguration in the presence of distributed generation. IEEE Trans Power Syst 28(1):317-325

[17] Zhang D, Fu ZC, Zhang LC (2008) Joint optimization for power loss reduction in distribution systems. IEEE Trans Power Syst 23(1):161-169

[18] Guimaraes MAN, Castro CA, Romero R (2010) Distribution systems operation optimization through reconfiguration and capacitor allocation by a dedicated genetic algorithm. IET Gener Transm Distrib 4(11):1213-1222

[19] Rosseti GJS, De Oliveira EJ, De Oliveira LW et al (2013) Optimal allocation of distributed generation with reconfiguration in electric distribution systems. Electr Power Syst Res 103:178-183

[20] Imran AM, Kowsalya M, Kothari DP (2014) A novel integration technique for optimal network reconfiguration and distributed generation placement in power distribution networks. Int J Electr Power Energy Syst 63:461-472

[21] Hung DQ, Mithulananthana N, Bansal RC (2013) A combined practical approach for distribution system loss reduction. Int $\mathrm{J}$ Ambient Energy 36(3):123-131
[22] Atwa YM, El-Saadany EF (2011) Probabilistic approach for optimal allocation of wind-based distributed generation in distribution systems. IET Renew Power Gener 5(1):79-88

[23] Yang XS (2011) Bat algorithm for multi-objective optimization. Int J Bio-Inspir Comput 3(5):267-274

[24] Sambariya DK, Prasad R (2014) Robust tuning of power system stabilizer for small signal stability enhancement using metaheuristic bat algorithm. Int J Electr Power Energy Syst 61:229-238

[25] Wang G, Guo LH, Duan H, et al. (2012) A bat algorithm with mutation for UCAV path planning. Sci World J 418946/15 pp

[26] Niknam T, Abarghooee RA, Zare M et al (2013) Reserve constrained dynamic environmental/economic dispatch: A new multiobjective self-adaptive learning bat algorithm. IEEE Syst J 7(4):763-776

[27] Sathya MR, Ansari MMT (2015) Load frequency control using bat inspired algorithm based dual mode gain scheduling of PI controllers for interconnected power system. Int J Electr Power Energy Syst 64:365-374

[28] Fister I, Rauter S, Yang XS et al (2015) Planning the sports training sessions with the bat algorithm. Neurocomputing 149-B:993-1002

[29] Kashi S, Minuchehr A, Poursalehi N et al (2014) Bat algorithm for the fuel arrangement optimization of reactor core. Ann Nucl Energy 64:144-151

[30] Rodrigues D, Pereira LAM, Nakamura RYM et al (2014) A wrapper approach for feature selection based on bat algorithm and optimum-path forest. Expert Syst Appl 41(5):2250-2258

[31] Fister I Jr, Fister D, Yang XS (2013) A hybrid bat algorithm. Elektrotehniski Vestnik 80(1/2):1-7

[32] Swarnkar A, Gupta N, Niazi KR (2011) A novel codification for meta-heuristic techniques used in distribution network reconfiguration. Electr Power Syst Res 81:1619-1626

[33] Yang XS (2013) Bat algorithm: Literature review and applications. Int J Bio-Inspir Comput 5(3):141-149

[34] Baran ME, Wu FF (1989) Network reconfiguration in distribution system for loss reduction and load balancing. IEEE Trans on Power Deliv 4(2):1401-1407

Neeraj KANWAR received the M. Tech. in Electrical Engineering (Power Systems) from Malaviya National Institute of Technology, Jaipur, India in 2010. She is presently perusing Ph.D. from the same institute. Her present research area is the application of AI techniques to power systems.

Nikhil GUPTA received M. Tech. and Ph.D., both in Electrical Engineering from Malaviya National Institute of Technology, Jaipur, India in 2006 and 2012, respectively. He is presently working as Assistant Professor in the same institute. His present research interests are the operation and control of power systems and computational intelligence.

K. R. NIAZI received M. E. in Electrical Engineering from JNV University, Jodhpur, India and in 1997, and Ph.D. in Electrical Engineering from Rajasthan University, Jaipur in 2003. He is with Malaviya National Institute of Technology, Jaipur, India since 1999. His areas of research interest are power system optimization, security analysis, distribution system optimization and application of ANN to power systems.

Anil SWARNKAR received M. Tech. and Ph.D., both in Electrical Engineering from Malaviya National Institute of Technology, Jaipur, India in 2005 and 2012, respectively. He is presently working as Assistant Professor in the same institute. His present research interests are operation and control of power systems and AI techniques. 